\title{
Causas de conversión en la cirugía laparoscópica de la enfermedad por reflujo gastroesofágico: análisis de nuestra experiencia
}

\author{
P. Priego, E. Lobo, A. Sanjuanbenito, E. Martínez Molina, J. Pérez de Oteyza, J. Ruiz Tovar, \\ G. Rodríguez Velasco y V. Fresneda
}

Servicio de Cirugía General y Digestivo. Hospital Ramón y Cajal. Madrid

\section{RESUMEN}

Introducción: desde su introducción en 1991, la cirugía antirreflujo por vía laparoscópica ha ido adquiriendo gran popularidad hasta convertirse en el procedimiento de elección de la enfermedad por reflujo gastroesofágico.

Objetivo: identificar y analizar de forma retrospectiva las causas de conversión del abordaje laparoscópico en la cirugía de la enfermedad por reflujo gastroesofágico y de la hernia de hiato.

Material y métodos: desde 1993 a agosto de 2007 se han efectuado en nuestro centro 606 cirugías antirreflujo por vía laparoscópica, encontrando 296 mujeres y 310 varones con una edad media de 53,3 años. La indicación fundamental fue la existencia de un reflujo gastroesofágico resistente al tratamiento médico con hernia de hiato asociada. De forma preoperatoria se van a efectuar estudios manométricos y pH-métricos, endoscopia oral y tránsito esofagogastroduodenal. La técnica quirúrgica de elección fue mayoritariamente la funduplicatura tipo Nissen-Rossetti.

Resultados: la estancia media postoperatoria fue de 2,7 días, realizándose conversión a cirugía abierta en 43 casos (7\%). Las conversiones fueron más frecuentes en la primera década de la curva de aprendizaje (26 vs. $17 \mathrm{p}<0,016$ ), y menores en el grupo de cirujanos expertos en cirugía laparoscópica avanzada (15 vs. 28, p < 0,017). En 17 casos la conversión fue debida a una complicación intraoperatoria y en 26 casos a dificultades técnicas. Las tasas de perforación esofágica y de neumotórax fueron del 0,8 y $1 \%$, mientras que la tasa de mortalidad y morbilidad fue del 0,1 y $12 \%$ respectivamente.

Conclusión: la tasa de conversión está dentro de los límites aceptables y ha disminuido con la experiencia.

Palabras clave: Cirugía laparoscópica antirreflujo. Conversión. Complicaciones intraoperatorias. Hernia de hiato.

\begin{abstract}
Background: since its introduction in 1991 laparoscopic antireflux surgery has gained great success and popularity among surgeons, and now it is the gold standard for the treatment of gastroesophageal reflux disease (GERD).

Aim: to identify and evaluate the causes of conversion in the laparoscopic surgery of GERD and hiatus hernia.

Material and methods: since January 1993 to August 2007 606 laparoscopic antireflux procedures were performed in our hospital. There were 296 women and 310 men with a median age of 53.5 years. The main indication for surgery was evidence of intractable or recurrent GERD symptoms after adequate medical treatment with associated hiatal hernia. The preoperative workup included manometry, pH-metry, oral endoscopy, and barium swallow. The surgical technique was mainly the Nissen-Rossetti procedure.

Results: mean postoperative hospital stay was 2.7 days. The operation had to be converted to an open procedure in 43 cases (7\%). Conversions were more frequent in the first decade of the learning curve (26 vs. 17, $\mathrm{p}<0.016)$, and fewer among the group of experts in advanced laparoscopic surgery (15 vs. 28, $p<0.017)$. In 17 cases conversions were due to an intraoperative complication whereas in 26 cases a conversion was done because of technical difficulties. Esophageal perforation and pneumothorax rates were 0.8 and $1 \%$, respectively, and mortality and morbidity rates were 0.1 and $12 \%$.
\end{abstract}

Conclusion: the rate of conversion is acceptable and significantly decreases with surgeon experience.

Key words: Laparoscopic antireflux surgery. Conversion. Intraoperative complications. Hiatus hernia.

Priego P, Lobo E, Sanjuanbenito A, Martínez Molina E, Pérez de Oteyza J, Ruiz Tovar J, Rodríguez Velasco G, Fresneda V. Causas de conversión en la cirugía laparoscópica de la enfermedad por reflujo gastroesofágico: análisis de nuestra experiencia. Rev Esp Enferm Dig 2008; 100: 263-267.

Recibido: 01-10-07.

Aceptado: 12-02-08.

Correspondencia: Pablo Priego Jiménez. C/ Fermín Caballero, 26, $1^{\circ} \mathrm{A}$. 16004 Cuenca.e-mail: papriego@hotmail.com

\section{INTRODUCCIÓN}

Desde su introducción en $1991(1,2)$ la cirugía antirreflujo por vía laparoscópica ha ido adquiriendo gran popu- 
laridad hasta convertirse en el procedimiento de elección de la enfermedad por reflujo gastroesofágico.

La experiencia adquirida durante estos años ha demostrado que la cirugía laparoscópica va a aportar mayores ventajas que desventajas, pues no sólo va a reducir el disconfort y el dolor postoperatorio, sino también acortar la estancia hospitalaria postoperatoria (3-9).

Sin embargo, la cirugía laparoscópica no está exenta de complicaciones (perforación gástrica y esofágica, estenosis del hiato esofágico, neumotórax, hemorragia,...) y en ocasiones requiere reintervenciones difíciles y de alto riesgo (10-13).

Lógicamente, estas complicaciones y conversiones a cirugía abierta van a disminuir conforme aumenta la experiencia del cirujano en la realización de procedimientos laparoscópicos.

El objetivo del trabajo es identificar y analizar las causas de conversión a cirugía abierta en la cirugía laparoscópica de la enfermedad por reflujo gastroesofágico y la hernia de hiato, basándonos en la experiencia adquirida en el Hospital Ramón y Cajal en los últimos 14 años.

\section{MATERIAL Y MÉTODOS}

Se efectúa un análisis retrospectivo entre enero de 1993 y agosto de 2007, incluyendo a 606 pacientes a los cuales se les realiza una cirugía antirreflujo por vía laparoscópica en el Hospital Ramón y Cajal. La cirugía fue realizada por un total de 30 cirujanos diferentes, incluyendo residentes. Para efectuar el análisis de los resultados vamos a dividir a los cirujanos en dos grupos en función del grado de experiencia quirúrgica en cirugía laparoscópica avanzada (expertos $v s$. no expertos).

La indicación principal para la cirugía fue la existencia de un reflujo gastroesofágico (RGE) resistente al tratamiento médico (IBP: inhibidores de la bomba de protones) junto con hernia de hiato asociada $(50,6 \%)$, realizándose colecistectomía simultáneamente en 59 pacientes (Tabla I).

El examen preoperatorio consta de: historia clínica y examen físico a todos los pacientes, endoscopia oral en 550 pacientes $(90,7 \%)$, tránsito esofagogastroduodenal en 489 pacientes $(80,7 \%)$, estudios manométricos y pHmétricos en 406 pacientes (67\%).

La técnica quirúrgica de elección fue mayoritariamente la funduplicatura tipo Nissen-Rossetti (Tabla II). El neumoperitoneo se crea habitualmente con aguja de Ve-

Tabla I. Indicaciones quirúrgicas

\begin{tabular}{lc}
\hline Indicación quirúrgica & Número de pacientes \\
\hline Hernia de hiato + RGE & 307 \\
Hernia de hiato & 111 \\
RGE que no cede con IBP & 90 \\
Complicaciones del RGE: & \\
Esofagitis grado III-IV & 57 \\
Esófago de Barrett & 34 \\
Estenosis esofágica & 7 \\
\hline
\end{tabular}

Tabla II. Técnicas quirúrgicas

\begin{tabular}{lc}
\hline Técnica quirúrgica & Número de pacientes \\
\hline Nissen-Rossetti & 458 \\
Toupet & 100 \\
Otros & 48 \\
Uso material protésico & 48 \\
\hline
\end{tabular}

ress en el hipocondrio izquierdo y trabajamos con presiones intraabdominales en torno a $12-14 \mathrm{mmHg}$. Generalmente utilizamos 5 trócares de $10 \mathrm{~mm}$ y la óptica suele ser de $0^{\circ}$, si bien esto puede ser modificado según los gustos de cada cirujano. En primer lugar efectuamos la disección del hiato esofágico, respetando siempre que sea posible las ramas hepáticas del nervio vago. En segundo lugar reducimos la hernia, si existe, y se diseca y aísla por completo el esófago (nos es útil la utilización de una cinta alrededor del esófago para facilitarnos la tracción del mismo). A continuación, siempre aproximamos los pilares diafragmáticos con sutura irreabsorbible de Ethibond (2-3 puntos) y se efectúa la funduplicatura (en general no somos partidarios de seccionar los vasos cortos, salvo que exista dificultad para el paso del fundus por detrás del esófago). En los casos de grandes defectos en el hiato esofágico, o en aquellos casos en los que la sutura de los pilares queda excesivamente tensa o los pilares son de mala calidad, colocamos una malla de polipropilenoPTFE (Crurasoft-BARD), fijada con 3-4 puntos de Ethibond a los pilares diafragmáticos.

Para el análisis estadístico de los resultados se utilizó el programa SPSS, analizando las variables cualitativas mediante el test de la Chi cuadrado y el de la probabilidad de Fisher. Se considera que el resultado es estadísticamente significativo cuando la $\mathrm{p}<0,05$.

\section{RESULTADOS}

De los 606 pacientes, encontramos 296 mujeres $(48,8 \%)$ y 310 varones $(51,2 \%)$, siendo la edad media de 53,3 años (rango 20-82 años). La estancia media hospitalaria postoperatoria fue de 2,7 días (rango 1-48 días).

La tasa de complicaciones ronda el $12 \%$ (72 casos en 606 pacientes) y la mortalidad es del $0,1 \%$ ( 1 caso).

En total, se van a producir 43 conversiones a cirugía abierta, siendo la tasa del 7\% (Tabla III).

Cuando comparamos la tasa de conversión en función de la curva de aprendizaje, observamos que esta es mayor (10\%: 26 casos en 260 pacientes) en la primera década (1993-2000) en comparación con la segunda década (2001-2007) (5\% 17 casos en 346 pacientes), siendo las diferencias estadísticamente significativas $(\mathrm{p}<0,016$; OR: $2,15$; intervalo de confianza del $95 \%$ entre $1,14-4,05)$.

Del mismo modo, cuando comparamos el número de conversiones en función de la experiencia laparoscópica del cirujano, observamos que estas son menores en el grupo de cirujanos expertos en cirugía laparoscópica avanzada 
Tabla III. Conversiones a cirugía abierta

\begin{tabular}{lc}
\hline Causas de conversión & Número de pacientes \\
\hline Dificultades en disección por exceso de grasa (obesidad) & 8 \\
Hemorragia & 7 \\
Hernia de hiato gigante no reducible & 6 \\
Adherencias secundarias a cirugía previa & 4 \\
Perforación gástrica & 4 \\
Complicaciones anestésicas & 3 \\
Perforación esofágica & 3 \\
Periesofagitis & 3 \\
Hepatomegalia & 3 \\
Imposibilidad de sutura de pilares & 2 \\
\hline
\end{tabular}

(15 casos en 405 cirugías) frente al grupo de cirujanos no expertos (28 casos en 201 cirugías), siendo las diferencias estadísticamente significativas ( $<<0,017$; OR: 0,45 ; intervalo de confianza del $95 \%$ entre $0,235-0,878$ ).

De acuerdo a la tabla III, observamos que la conversión a cirugía abierta se debe en 26 de las ocasiones a dificultades técnicas (dificultades en disección por exceso de grasa, hernia de hiato gigante no reductible, adherencias secundarias a cirugía previa, imposibilidad de sutura de pilares, hepatomegalia y periesofagitis) y en 17 casos se debe a complicaciones intraoperatorias que no pueden resolverse por vía laparoscópica (hemorragia, perforación gástrica y esofágica; y complicaciones anestésicas).

Cuando realizamos el análisis comparativo de las causas de conversión por décadas (Tabla IV) observamos que las principales causas de conversión van a ser similares en ambos grupos (dificultades en disección por exceso de grasa y hemorragia), encontrando únicamente diferencias estadísticamente significativas en las perforaciones gástricas, que van a ser mayores en la primera década $(\mathrm{p}<0,033)$.

Sin embargo, no siempre que se produjo una complicación intraoperatoria hubo que convertir a cirugía abierta (Tabla V).

De los 43 casos en los que hubo que convertir a cirugía abierta, la indicación quirúrgica fue hernia de hiato asociada a reflujo gastroesofágico en 21 casos $(48,8 \%)$, hernia de hiato gigante en 19 casos $(44,2 \%)$ y esófago de Barrett en 3 casos (7\%). Si desglosamos las indicaciones quirúrgicas que condujeron a la conversión por décadas, observamos que la causa más frecuente de conversión en la primera década fue la combinación de hernia de hiato y reflujo gastroesofágico (61,5\%: 16 de 26 casos; $p<0,007$;
Tabla V. Complicaciones intraoperatorias

\begin{tabular}{lcc}
\hline Complicación & Tratamiento & \\
& Laparoscópica \\
\hline Apertura gástrica: 15 casos & 4 & 11 \\
Apertura pleural: 6 casos & & 6 \\
Hemorragia: 16 casos & 7 & 9 \\
Hematoma hepático: 2 casos & & 2 \\
Enfisema subcutáneo: 2 casos & & 2 \\
\hline
\end{tabular}

OR: 3,77; intervalo de confianza del $95 \%$ entre 1,36$10,5)$, mientras que en la segunda década fue la hernia de hiato gigante (59\%: 10 de 17 casos; $p<0,024$; OR: 3,091; intervalo de confianza del $95 \%$ entre $1,12-8,5$ ).

\section{DISCUSIÓN}

Desde su introducción en $1991(1,2)$ la cirugía antirreflujo por vía laparoscópica ha ido adquiriendo gran popularidad hasta convertirse en el procedimiento de elección de la enfermedad por reflujo gastroesofágico.

En la figura 1, que representa nuestra casuística, observamos en primer lugar un aumento exponencial del número de cirugías antirreflujo laparoscópicas desde 1993 donde no llega a 10 pacientes, hasta el año 2007, en donde se sobrepasan los 50 pacientes. Este incremento ha sido debido en parte a los grandes éxitos conseguidos por la cirugía (con tasas del 90\% libre de enfermedad 10 años después de la cirugía) (14-16) y sobre todo a las ventajas ofrecidas con el abordaje laparoscópico.

Sin embargo, la cirugía laparoscópica no está exenta de complicaciones y a veces requiere reintervenciones

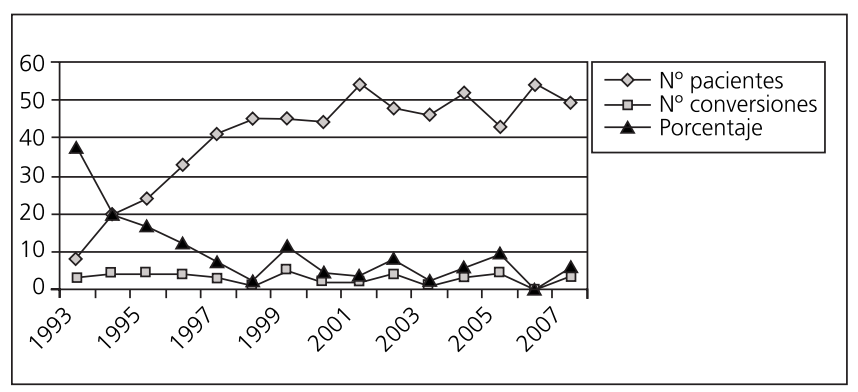

Fig. 1.

Tabla IV. Causas de reconversión en función de la curva de aprendizaje

\begin{tabular}{ll}
\hline $1993-2000$ & $2001-2007$ \\
\hline Dificultades en disección por exceso de grasa: 5 casos (19\%) & Dificultades en disección por exceso de grasa: 3 casos (17,6\%) \\
Hemorragia: 4 casos $(15,4 \%)$ & Hemorragia: 3 casos (17,6\%) \\
Perforación gástrica: 4 casos $(15,4 \%)$ & Adherencias secundarias a cirugía previa: 2 casos \\
Hernia de hiato no reducible: 3 casos & Imposibilidad de cierre de pilares: 2 casos \\
Hepatomegalia: 2 casos & Hepatomegalia: 1 caso \\
Periesofagitis: 2 casos & Periesofagitis: 1 caso \\
Adherencias secundarias a cirugía previa: 2 casos & Perforación esofágica: 1 caso \\
Perforación esofágica: 2 casos & Complicaciones anestésicas: 1 caso \\
Complicaciones anestésicas: 2 casos &
\end{tabular}


quirúrgicas difíciles y de alto riesgo (3-13). Afortunadamente, en nuestra experiencia el número de complicaciones $(12 \%)$ no es muy alto y sólo se produjo un fallecimiento $(0,1 \%)$, secundario a una embolia pulmonar en un paciente obeso mórbido, y en el cual hubo que convertir la cirugía laparoscópica por resultar difícil la disección, debido a un exceso de grasa intraabdominal.

En cuanto a la tasa de conversión a cirugía abierta, en nuestra experiencia es del 7\%. Esta, pese a realizarse por numerosos cirujanos no dedicados de forma exclusiva a la cirugía laparoscópica, está dentro de los límites aceptables descritos por otros autores (17-20) y ha disminuido con la experiencia. Así, en la figura 1 vamos a observar una disminución gradual del porcentaje de conversiones a cirugía abierta, de manera que en la primera década de la curva de aprendizaje (1993-2000) era del 10\%, frente al 5\% actual (2001-2007). El papel de la curva de aprendizaje resulta por tanto fundamental en la mejora de los resultados y en la disminución del número de conversiones. De acuerdo a esta afirmación, en nuestra serie se observa un menor número de conversiones a cirugía abierta dentro del grupo de cirujanos expertos en cirugía laparoscópica avanzada frente a los no expertos (15 vs. 28). Sin embargo, debemos tener en cuenta que la conversión a una cirugía abierta no implica el fracaso del cirujano y que esta no es exclusiva de los cirujanos no expertos, recordando que el objetivo de la intervención es en definitiva el tratamiento del reflujo y no el efectuar un procedimiento laparoscópico a toda costa (18).

La mayor parte de los estudios considera que el número mínimo a partir del cual un cirujano comienza a adquirir destreza y experiencia en el manejo de esta cirugía y empieza a disminuir la tasa de conversión y complicaciones está en torno a los 20 pacientes (21-24). Aun así, creemos que la cirugía laparoscópica del reflujo gastroesofágico, a pesar de considerarse dentro del grupo de cirugías laparoscópicas avanzadas, debería ser un procedimiento quirúrgico que el residente tendría que efectuar al menos una vez durante su periodo de formación, ayudado por un personal del staff experimentado.

En términos generales, y al igual que Collet y Cadiere (18), vamos a dividir las causas de conversión a cirugía abierta en dos grandes grupos:

1. Dificultades técnicas: conjunto de circunstancias que interfieren en el normal desarrollo de la cirugía y que hacen que el cirujano no se sienta a gusto en el campo operatorio, p. ej. obesidad, adherencias de cirugías previas, lóbulo hepático izquierdo de gran tamaño y que interfiere en la correcta disección del hiato,...

Consideramos que, para que un procedimiento laparoscópico se convierta a cirugía abierta, no es imprescindible que exista una complicación intraoperatoria, sino que también estaría indicado en aquellas situaciones en las que, tras un largo periodo de disección (más de 1 hora), la operación no ha progresado de manera adecuada.

Dos circunstancias que favorecen el que la cirugía se desarrolle de forma correcta son una buena introducción de los trócares de laparoscopia, adaptados a la morfología del paciente y una correcta exposición del área hiatal.

2. Complicaciones intraoperatorias: que en función de la experiencia del cirujano se pueden solucionar a través de un abordaje laparoscópico o bien requerirá una conversión a un procedimiento abierto, p. ej. perforación esofágica y gástrica, presencia de sangrado persistente que impide una correcta visualización del campo quirúrgico,...

Si analizamos más detalladamente nuestra casuística, observamos que las causas más frecuentes de conversión a cirugía son: dificultades en disección por exceso de grasa (8 casos), hemorragia (7 casos) y hernia de hiato gigante no reductible (6 casos).

La división de las causas de conversión por décadas señala que las causas son similares entre ambos grupos, con la salvedad de la perforación gástrica, que supone la tercera causa de conversión a cirugía abierta con 4 casos en la primera década frente a ningún caso en la segunda década. La explicación sería la mayor experiencia del cirujano en la realización de suturas laparoscópicas y al manejo más cuidadoso del estómago con el material quirúrgico (babcoks).

En cuanto al análisis de las conversiones en función de la indicación quirúrgica, únicamente cabe reseñar que, en la actualidad, y como parece lógico, el mayor número de conversiones se realiza en pacientes con hernias de hiato gigantes (paraesofágicas o mixtas), dada la gran dificultad que presentan a la hora de manipularlas y reducirlas, si bien esto no supone una contraindicación para el abordaje laparoscópico en el momento actual.

Del conjunto de complicaciones intraoperatorias que condicionan la conversión a una cirugía abierta, destaca la presencia de la hemorragia, sin embargo, y a pesar de que esta se ha producido con cierta magnitud en 16 pacientes $(2,6 \%)$, sólo ha sido la causa de conversión en 7 casos $(1 \%)$. En este punto consideramos de gran utilidad tanto la disección y coagulación con el bisturí ultrasónico, como el uso de gasas para conseguir el cese del sangrado.

Probablemente la complicación más importante por su gravedad en la cirugía antirreflujo vía laparoscópica sea la perforación esofágica ( $26 \%$ de mortalidad), con tasas que oscilan en torno al $1 \%$ en las grandes series de la literatura (25). En nuestra serie se ha producido en 5 casos $(0,8 \%)$, aunque sólo ha sido necesaria la conversión en 3 de ellos $(0,5 \%)$. En uno de los casos se produjo desafortunadamente una perforación del esófago torácico al pasar la bujía de calibración (en una paciente con una estenosis esofágica tratada previamente con dilataciones), por lo que hubo que efectuar una toracotomía para resolver la perforación. Aun así, afortunadamente, esta no ha sido causante de ningún caso de muerte, aunque ha añadido no sólo un aumento de la morbilidad, sino también una mayor estancia hospitalaria.

Otra complicación frecuente en cirugía laparoscópica es la apertura accidental de la cavidad gástrica, sobre todo con la manipulación de las pinzas de agarre o babcocks. En nuestra experiencia, esto ha ocurrido en 15 casos, pudien- 
do resolverse en 11 de ellos por un abordaje laparoscópico y teniendo que convertir a 4 pacientes $(0,6 \%)$.

Por último, la apertura de la pleura durante la disección del hiato esofágico ocurrió en 6 pacientes (1\%), sobre todo en aquellas hernias de hiato paraesofágicas-mixtas con gran saco herniario. Sin embargo, en ninguno de los casos fue necesaria la colocación de un tubo de tórax.

Por tanto, y para concluir, creemos que el abordaje laparoscópico es, en la actualidad, de elección en la cirugía del hiato esofágico. La incidencia de complicaciones intraoperatorias en nuestra serie ha sido baja y fundamentalmente en la etapa inicial (curva de aprendizaje). La tasa de conversión, pese a realizarse por numerosos cirujanos no dedicados de forma exclusiva a la cirugía laparoscópica, está dentro de los límites aceptables y ha disminuido con la experiencia.

\section{BIBLIOGRAFÍA}

1. Geagea T. Laparoscopic's Nissen's fundoplication: Preliminary report on ten cases. Surg Endosc 1991; 5: 170-3.

2. Dallegmane B, Weerts JM, Jehaes C, et al. Laparoscopic Nissen fundoplications: Preliminary report. Surg Laparosc Endosc 1991; 1: 138-43.

3. Horgan S, Pellegrini CA. Surgical treatment of gastroesophageal reflux disease. Surg Clin North Am 1997; 77: 1063-82.

4. Trullenque S, Torres T, Martí E, et al. Cirugía de la enfermedad por reflujo gastroesofágico: estudio comparativo entre los abordajes abierto y laparoscópico. Rev Esp Enferm Dig 2005; 97 (5): 328-37.

5. Kamholz T, Bammer T, Wykypiel HJ, Pasiut M, Pointner R. Quality of life and surgical outcome after laparoscopic Nissen and Toupet fundoplication: One year follow up. Endoscopy 2000; 32: 363-8.

6. Salminen PT, Laine SO, Ovaska JT. Late subjective results and symptomatic outcome after laparoscopic fundoplication. Surg Laparosc Endosc Percutan Tech 2006; 16: 203-7.

7. Anvari M, Allen C. Five year comprehensive outcomes evaluation in 181 patients after laparoscopic antireflux procedures. Surg Endosc 2002; 16: 753-7.

8. Booth MI, Jones L, Stratford J, Dehn TC. Results of laparoscopic Nissen fundoplication at 2-8 years after surgery. Br J Surg 2002; 89: 476-81.

9. Zaninotto $\mathrm{G}$, Portale $\mathrm{G}$, Costantini M, et al. Long term results (6-10 years) of laparoscopic fundoplication. J Gastrointest Surg 2007; 11
(9): 1138-45.

10. Pessaux P, Arnaud JP, Ghavami B, et al. Morbidity of laparoscopic fundoplication for gastroesophageal reflux: A retrospective study about 1470 patients. Hepatogastroenterology 2002; 49: 447-50.

11. Dallegmane B, Weerts JM, Jehaes C, Markiewiecz. Causes of failures of laparoscopic antirreflux operations. Surg Endosc 1996; 10: 305-10.

12. Hunter JG, Smith CD, Branum GD, Waring JP, Trus TL, Cornwell $\mathrm{M}$, et al. Laparoscopic fundoplication failures: Patterns of failure and response to fundoplication revision. Ann Surg 1999; 230: 595-604

13. Soper NJ, Dunnegan D. Anatomic fundoplication failure after laparoscopic antireflux surgery. Ann Surg 1999; 229: 669-76.

14. Dallegmane B, Weerts JM, Markiewicz S, Dewandre JM, Wahlen C, Monami B, et al. Clinical results of laparoscopic fundoplication at ten years after surgery. Surg Endosc 2006; 20 (1): 159-65.

15. Lafullarde T, Watson DI, Jamieson GG, Myers JC, Game PA, Devitt PG. Laparoscopic Nissen fundoplication: Five years results and beyond. Arch Surg 2001; 136: 180-4.

16. Pessaux P, Arnaud JP, Delattre JF, Meyer C, Baulieux J, Mosnier H Laparoscopic antirreflux surgery. Five year results and beyond in 1340 patients. Arch Surg 2005; 140: 946-51.

17. Dallegmane B, Weerts JM, Jeahes C, et al. Results of laparoscopic Nissen fundoplication. Hepatogastroenterology 1998; 45: 1338-43.

18. Collet D, Cadiere GB. Conversions and complications of laparoscopic treatment of gastroesophageal reflux disease. Am J Surg 1995; 169: 622-6.

19. Coelho JCU, Wiederkehr JC, Campos ACL, Andrigueto PC. Conversions and complications of laparoscopic treatment of gastroesophageal reflux disease. J Am Coll Surg 1999; 189: 356-61.

20. Zaninotto G, Molena D, Ancona E. A prospective multicenter study on laparoscopic treatment of gastroesophageal reflux disease in Italy. Surg Endosc 2000; 14: 282-8.

21. Watson DI, Baigrie RJ, Jamieson GG. A learning curve for laparoscopic fundoplication. Definable, avoidable or waste of time? Ann Surg 1996; 224: 198-203.

22. Salminen P, Hiekkanen H, Laine S, Ovaska J. Surgeons' experience with laparoscopic fundoplication after the early personal experience: Does it have an impact on the outcome? Surg Endosc 2007; 21: 1377 82.

23. Hwang H, Turner LJ, Blair NP. Examining the learning curve of laparoscopic fundoplications at an urban community hospital. Am J Surg 2005; 189: 522-26; discussion 526.

24. Voitk A, Joffe J, Álvarez C, Rosenthal G. Factors contributing to laparoscopic failure during the learning curve for laparoscopic Nissen fundoplication in a community hospital. J Laparoendosc Adv Surg Tech A 1999; 9: 243-8.

25. Schauer PR, Meyers WC, Eubanks S, et al. Mechanisms of gastric and esophageal perforations during laparoscopic Nissen fundoplication. Ann Surg 1996; 223: 43-52. 